Performance 2002, IFIP Int. Symposium on Computer Performance Modeling, Measurement and Evaluation,

Rome, Italy, September 2002.

\title{
Capturing the spatio-temporal behavior of real traffic data *
}

\author{
Mengzhi Wang, Anastassia Ailamaki, and Christos Faloutsos \\ Carnegie Mellon University, Pittsburgh, PA 15213 \\ \{mzwang, natassa, christos\}@cs.cmu.edu
}

\begin{abstract}
Traffic, like disk and memory accesses, typically exhibits burstiness, temporal locality and spatial locality. There is much recent ground-breaking work on temporal modeling (self-similarity etc), on disk and web traffic, with several statistical models that generate realistic series of timestamps. However, no work generates realistic traces for both time and location (eg., block-id). In fact, except for qualitative speculations, it is not even known whether/how the time-stamps are correlated with the locations, nor how to measure this correlation, let alone how to re-produce it realistically.

These are exactly the problems we solve here: (a) We propose the 'entropy plots' to quantify the spatial/temporal correlation (or lack of it), and (b) we propose a new model, the 'PQRS' model, that captures all the characteristics of real spatio-temporal traffic. Our model can generate traffic that is bursty (or uniform) on time; bursty or uniform on space; and it can mimic the correlation between space and time, whenever such correlation exists. Moreover, it requires very few parameters ( $p, q, r$, and the grand total of disk/memory accesses); and it has linear scalability in computing these parameters. Experiments with multiple real data sets (disk traces from HP Labs, TPC-C memory traces), show that our model can mimic real traces very well, while the only obvious alternative, the independence assumption, leads to more than $60 x$ worse error.
\end{abstract}

*This material is based upon work supported by the National Science Foundation under Grants No. IIS-9988876, IIS-0083148, IIS-0113089, and by the Defense Advanced Research Projects Agency under Contract N66001-00-1-8936. Additional funding was provided by donations from Intel. Any opinions, findings, and conclusions or recommendations expressed in this material are those of the author(s) and do not necessarily reflect the views of the National Science Foundation, DARPA, or other funding parties. 


\section{Introduction}

Modeling traffic data, such as disk I/O, memory accesses, web and LAN traffic, is vital for performance evaluation studies. A simple and accurate statistical model has several advantages: (a) We can run 'what if' scenarios, by generating as long or as short a trace as we want; or by varying the load, burstiness and other parameters of our statistical model; (b) We need much less space: a real disk/memory/network trace may take huge space; a statistical model typically requires only a handful of parameters; (c) We can do analytical performance studies: For example, if we know that our traffic is Poisson, we can estimate analytically queue length distributions at a server with a given service time distribution.

Previous attempts on traffic modeling focus mainly on the temporal aspects. Location information, another important dimension, is usually left out of the picture even though the service time of a request depends on both the arrival time and the location. This work takes both time and location into consideration. In particular, we would like to answer the following questions:

- What is the spatial behavior the traces? Are all the disk blocks equi-probable (i.e. random accesses in credit card applications) or should we expect a Gaussian/Poisson disk of requests on each cylinder? Or maybe piece-wise uniform?

- What is the spatio-temporal correlation? Should we worry about the issue? How close to reality is the (convenient) independence assumption?

- The hardest one of all is to develop a statistical model that will naturally capture burstiness, uniformity, and correlation? A mixture of 2-dimensional Gaussian or Marked Point Processes (if so, with what arrival rates)?

More specifically, we want to find a model to generate a realistic trace that has the same temporal and spatial behavior as the real one.

Problem 1 Given a two-dimensional trace, $Y=\{(t, s)\}$, (i.e. $(t, s)$ defines a request of arrival time $t$ on location s.), develop a mathematical model that can generate a synthetic trace, $Y^{\prime}=$ $\left\{\left(t^{\prime}, s^{\prime}\right)\right\}$, that has "similar" spatio-temporal behavior as $Y$.

The goodness of the model can be evaluated by comparing the synthetic traces to the real traces in terms of both statistical measures (i.e. mean, burstiness, and correlation) and performance 
behavior (i.e. response time distributions for disk traces). The latter, in our opinion, is more important for practical reasons.

Compactness and efficiency of the model are two additional concerns. A naive model can simply remember the given trace and reproduce it as a synthetic trace when required, but this hardly saves any space or effort, nor allows for generation of longer traces. The ideal model should (a) require few parameters, (b) exhibit burstiness over time and space, (c) preserve the spatio-temporal correlation, and (d) have linear scalability.

The paper is organized as follows. Section 2 reviews the related work. Section 3 studies the behavior of the real world traffic and Section 4 provides a measure for both the burstiness and the correlation. Section 5 introduces the PQRS model. Section 6 evaluates the model using real memory traces. Section 7 concludes the work and comments on future related research directions.

\section{Related Work}

We distinguish three lines of work in traffic modeling. Most of the previous work focused on network traffic. Disk I/O are usually generated by mixing sequential and random accesses [15]; however, it involves several parameters, which are hard to determine. Therefore, generating realistic disk traces is still an unsolved problem [8].

Temporal models. The discovery of self-similarity and burstiness in network traffic invalidates the classical traffic modeling works based on Poisson assumption [11]. Various statistical models, such as fractal ARIMA[9], Multifractal Wavelets[12], and b-model[16], have been proposed to capture the temporal burstiness.

Spatial models. Approaches based on spatial statistics models [6] generally assume that the data is (multivariate) Gaussian, which produces smooth traffic data, therefore, is inappropriate for the burty traffic. Marked point processes [5] can also be used to model the occurrence of events in time and space. However, these models typically require some kind of structural assumptions on the underlying intensity, and choosing this structure in general is a non-trivial problem. The randomized version of the PQRS model we introduce in this paper can be thought of as specifying the intensity of a marked point process model for the data. 

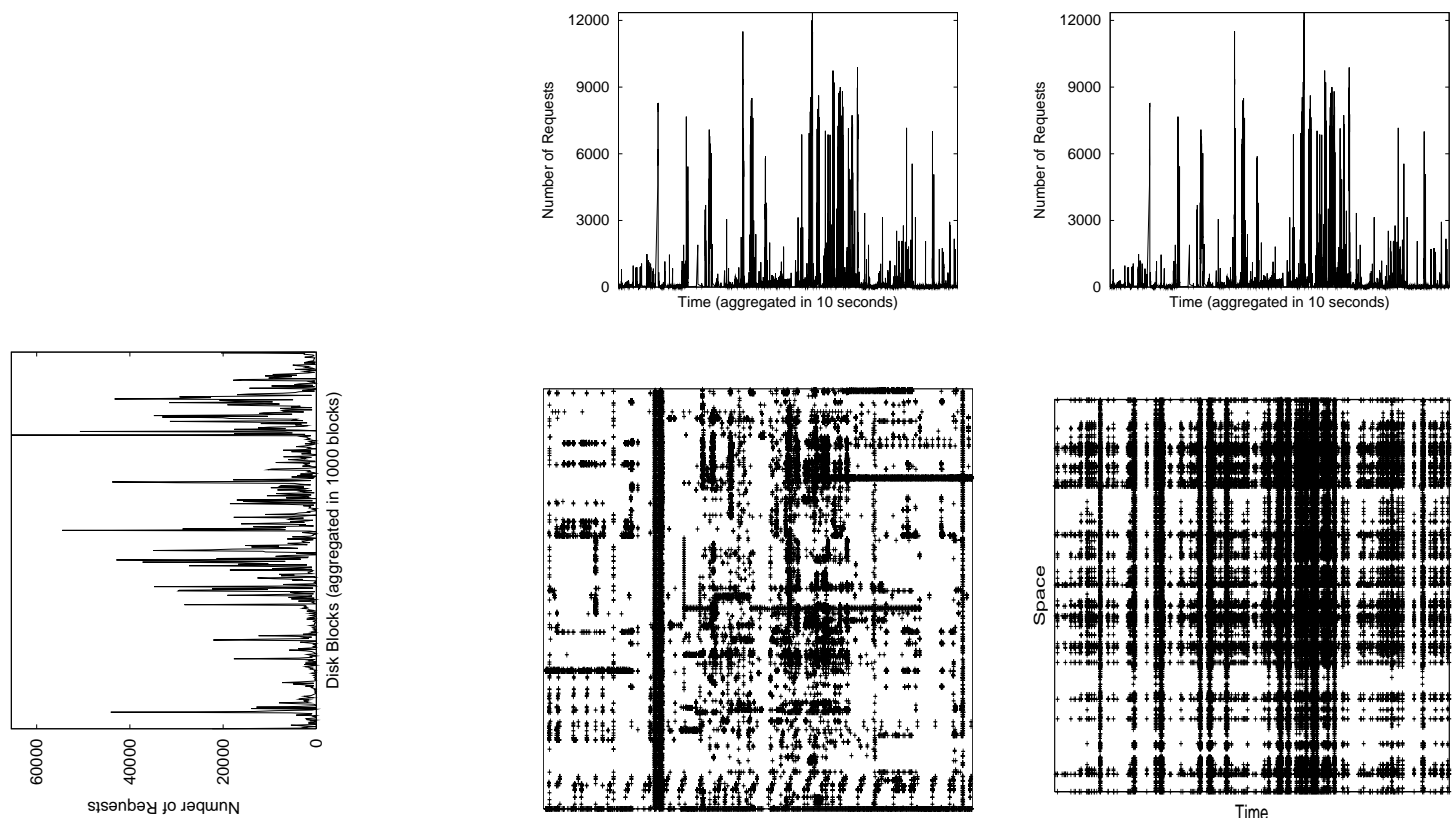

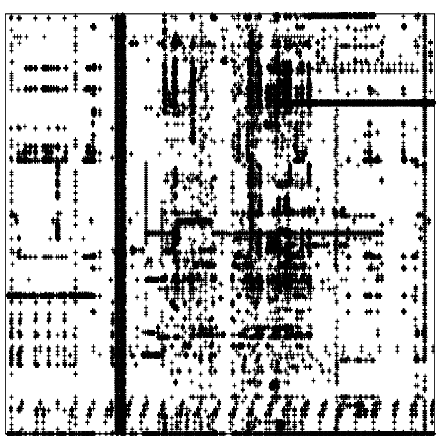

(a) A sample disk trace

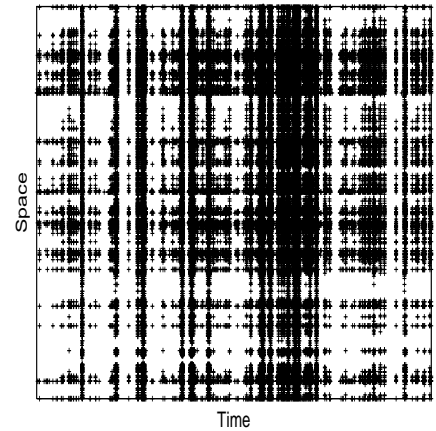

(b) The i-model trace

Figure 1: Time-space plot of the sample disk trace and the i-model trace

Physical models. Another approach tackles the problem by simulating user behavior. The ON/OFF model in the SURGE web traffic generator [2] aggregates requests from multiple users where the user thinking time follows a heavy-tailed distribution. The model is able to produce twodimensional traces, but it's unclear if the spatio-temporal correlation is well preserved in synthetic traces.

Our approach takes traffic modeling work one step further by modeling both the spatio-temporal behavior. The PQRS model is the first model that not only captures bursty behavior along both space and time, but accurately captures the spatio-temporal correlation.

\section{Observing Data: Naive Model}

Understanding the real traffic data is crucial before we can start to build a model. This section studies the behavior of the real world traffic data and introduces an initial attempt, the i-model. 


\subsection{Burstiness}

Define the time-space plot as the projection of trace $Y$ to the time space plane. Figure 1 shows the time-space plot for the cello disk trace [13]. $C_{T, S}(t, s)$ is the number of tuples $(t, s)$ in the trace. That is, the number of requests of arrival time $t$ of location $s$. Further projection of the trace onto time and space gives the "marginal" one-dimensional traces $C_{T}$ and $C_{S}$, in which $C_{T}(t)$ and $C_{S}(s)$ tell the number of requests at time $t$ or on block $s$. We observe "bursty" behavior (i.e. non-uniformity) in both marginals.

- Temporal burstiness. The temporal burstiness is expected: various traffic data, such as disk I/O traffic [10] and network traffic [11, 7], have all been shown bursty.

- Spatial burstiness. $C_{S}$ is apparently not uniform, nor piece-wise uniform as some previous work assumes. The spatial skewness has been noticed before [4], yet there is little efforts on modeling it. Existing one-dimensional models should be able to capture the spatial burstiness in the same way as they do for the temporal burstiness since the bursty behavior looks similar for time and space.

However, even if we do know both marginals, we can not generate two-dimensional traces if no combining algorithm is available. The straight-forward combining algorithm is the i-model, which is discussed in the next section.

\section{$3.2 \quad$ I-Model}

The i-model generates a two-dimensional trace by "multiplying" two marginal traces. For example, if $10 \%$ of the total requests arrive between at time $t$ and $5 \%$ of the total requests occur on disk blocks on $s, 10 \% \times 5 \%$ of the total requests arrive at $t$ on disk block number $s$.

Formally, the I-model specifies that given $C_{T}$ and $C_{S}$,

$$
C_{T, S}(t, s)=C_{T}(t) \times C_{S}(s) / M, \quad t=1,2, \ldots ; \quad s=1,2, \ldots ;
$$

where $M$ is the total number of requests in the trace.

The i-model works with all one-dimensional models and preserves the temporal and spatial burstiness because the marginals of the generated two-dimensional trace are exactly the same as 
the original. In addition, it requires no parameters. Despite of numerous advantages, the i-model ignores a very important property of the traffic: strong spatio-temporal correlation. Figure 1 (b) shows a two-dimensional trace generated by the i-model with $C_{T}$ and $C_{S}$ derived from the real trace. We observe significant differences between the i-model trace and the real one. The difference is attribute to the existence of strong spatio-temporal correlation in the real trace. In fact, the independence assumption leads to grossly pessimistic results, as we show in Section 6 .

\section{Proposed Method to Quantify Burstiness}

The i-model contradicts the intuition that requests arriving closely in time tend to access nearby objects. Thus, the correlation will have a great impact on the performance behavior because requests to nearby objects take less time to serve. This section discusses how to measure the correlation.

\subsection{Definitions}

Various measures are proposed to measure the uniformity of a probability function, such as gini index and entropy. We employ the entropy as our measurement in this paper. (The parameters used in the paper along with their definitions are summarized in Table 1.)

Entropy is a well-known concept in information theory to measure the uniformity of a discrete probability function [14]. Recall that entropy on a random variable $E$, (e.g. disk block id of random requests), is defined as

$$
H(E)=-\sum_{i=0}^{N} p_{i} \log _{2} p_{i},
$$

where $p_{i}$ is the probability that event $E_{i}$ will happen (e.g. the $i$-th block will be hit) and $N$ is the total number of possible outcomes (e.g. total number of disk blocks). $H$ is close to 0 if the distribution is highly skewed while a uniform distribution gives the maximum value of $\log _{2} N$ for $H$.

The joint entropy on two random variables is defined similarly: for a given probability function $P=\left\{p_{i, j}\right\}$ on two random variables $\{E\}$ and $\{F\}$, (e.g. arrival time and disk block id of random requests), where $p_{i, j}$ gives the probability that both event $E_{i}$ and event $F_{j}$ will happen, (e.g. a disk 


\begin{tabular}{|c|l|}
\hline$P_{T, S}(t, s)$ & Probability that a request on location $s$ will arrive at time $t$. \\
\hline$P_{T}(t)$ & Probability that a request will arrive at time $t$. \\
\hline$P_{S}(s)$ & Probability that a request is on location $s$ \\
\hline$H(E)$ & Entropy of a random variable $E$ \\
\hline$H_{T}^{(n)}$ & Temporal entropy at aggregation level $n$ \\
\hline$R_{T}$ & Slope of the temporal entropy plot \\
\hline$H_{S}^{(n)}$ & Spatial entropy at aggregation level $n$ \\
\hline$R_{S}$ & Slope of the spatial entropy plot \\
\hline$H_{T, S}^{(n)}$ & Joint entropy on time and space at aggregation level $n$ \\
\hline$R_{T, S}$ & Slope of the joint entropy plot \\
\hline$(p, q, r, s)$ & Parameters to the PQRS model \\
\hline$M$ & Total number of requests in a trace \\
\hline
\end{tabular}

Table 1: Symbols table.

request at block id $j$ will arrive at $i$ ), the joint entropy on $E$ and $F$ is defined as

$$
H(E, F)=-\sum_{i, j} p_{i, j} \log _{2} p_{i, j}
$$

Definition 1 The mutual information $I(E ; F)$ on two random variables $E$ and $F$ is defined as

$$
I(E ; F)=H(E)+H(F)-H(E ; F) .
$$

The mutual information $I(E ; F)$ indicates the degree of correlation between $E$ and $F$. It becomes zero if $E$ and $F$ are independent.

\subsection{Entropy Plots}

We can apply the above definitions to traces to measure the burstiness and spatio-temporal correlation. The question is, then, what the granularity should be. If we calculate the entropy on the finest resolution, the mutual information on time and space will be very close to zero because no correlation will be observed. Our answer is to calculate the entropy values at all "aggregation" levels.

To find the entropy values at aggregation level $n$, the trace is divided into $2^{n} \times 2^{n}$ grids in the time-space plot. Figure 2 (a) shows the grids at aggregation level $n=2 . P_{T, S}^{(n)}$ is the probability 


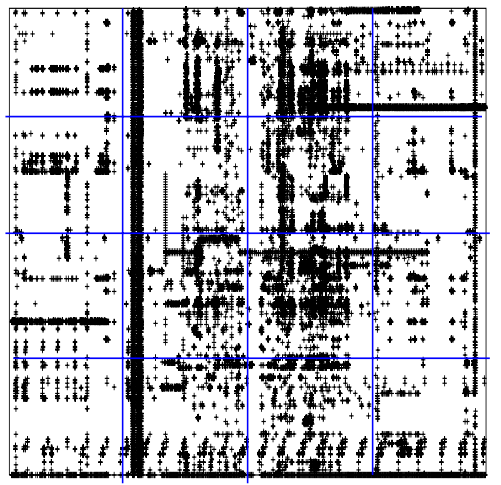

(a) Aggregation level $2\left(2^{2} \times 2^{2}\right.$ grids)

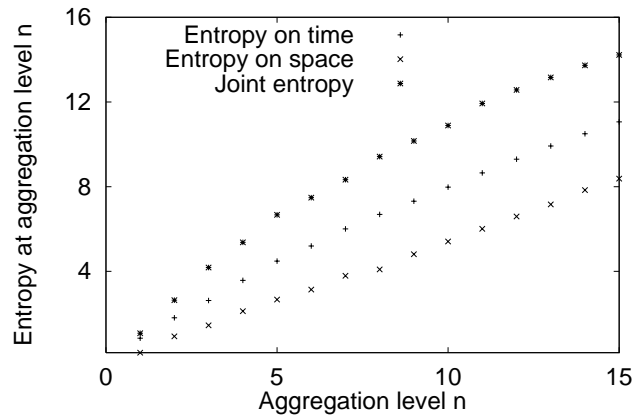

(b) Entropy plots for the sample disk trace.

Figure 2: Entropy plot.

function that gives the probability that a request will fall into each grid, i.e. a request on location $\left(s_{1}, s_{2}\right)$ arriving at time $\left(t_{1}, t_{2}\right) . P_{T}^{(n)}$ and $P_{S}^{(n)}$ are the projections of $P_{T, S}^{(n)}$ on time and space and can be easily derived from the given trace.

Definition 2 At aggregation level $n$, define the entropy on time, space and the joint entropy for a given trace as

$$
\left\{\begin{array}{l}
H_{T}^{(n)}=H\left(P_{T}^{(n)}\right) \\
H_{S}^{(n)}=H\left(P_{S}^{(n)}\right) \\
H_{T, S}^{(n)}=H\left(P_{T, S}^{(n)}\right) .
\end{array}\right.
$$

Then, the entropy plots are the plots of the entropy values against the aggregation level $n$.

The entropy plot provides an insight on how the burstiness and correlation change across different resolution levels. The points form a line when the burstiness and correlation are stable at different granularities. Surprisingly, real traffic shows stable burstiness and correlation over aggregation as the linear entropy plots of the sample disk trace suggest (Figure 2 (b)).

Lemma 1 For a trace of stable temporal and spatial burstiness and spatio-temporal correlation, all the entropy plots are linear:

$$
\left\{\begin{array}{l}
H_{T}^{(n)}=n R_{T} \\
H_{S}^{(n)}=n R_{S} \\
H_{T, S}^{(n)}=n R_{T, S}
\end{array}\right.
$$


The intuition behind $R_{T}$ is the rate of information contained in one more bit of time-stamp.

- When all the requests come in a burst, all the time-stamps will be the same and the all the bits are useless, which leads to $R_{T}=0$.

- When the requests are uniformly distributed along time, all the bits in the time-stamps are useful and $R_{T}$, in this case, is 1.

Similarly, $R_{S}$ gives the rate of information in the location bit. Denote $R_{T}+R_{S}-R_{T, S}$ as $R_{I}$. $R_{I}$ tells the mutual information per bit, e.g. how much information the time-stamp bit tells about the location of the request.

- When $R_{I}$ equals to 0 , the time-stamp and the location of a request is independent.

- The real traffic data shows strong spatio-temporal correlation. The real trace in Figure 2 (b) gives $0.722,0.573,0.881$ as the estimated values for $R_{T}, R_{S}$, and $R_{T, S}$. The calculated value of $R_{I}$ turns out to be 0.414 , indicating strong spatio-temporal correlation. The i-model trace, on the other hand, render 0.001 for $R_{I}$, which suggests independence between time and space. (Hence the name i-model.)

\section{Proposed Model: PQRS Model}

The i-model fails to capture the spatio-temporal correlation in real traffic. The following sections present a new two-dimensional model, called the "PQRS" model, which has intrinsically stable burstiness and correlation.

\subsection{Generation: PQRS Model}

The PQRS model generates a two-dimensional trace using four parameters, namely, $p, q, r, s$, where $p+q+r+s=1$. The recursive construction is the reverse process of the entropy plot aggregation as illustrated in Figure 3 (a). At first, the probability that a request will fall into the square is 1 . In step 1 , the time-space plot is divided into $2 \times 2$ grids and the probability 


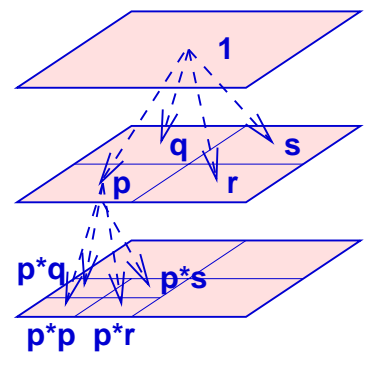

(a) Generation of a PQRS model

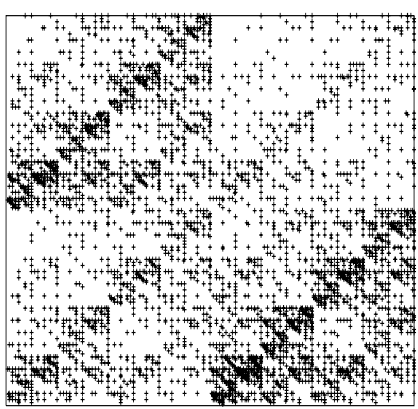

(b) A Sample PQRS trace

Figure 3: Recursive trace generation process for the PQRS model.

that a request falls in each grid is $p, q, r, s$ respectively. In step 2, each grid is further divided into 4 small grids and the requests in the grid are distributed to the four small grids with the same ratio, $p, q, r, s$. The process goes on recursively until the required resolution on time and space is achieved. Figure 3 (b) gives a sample trace generated by the PQRS model with $p, q, r, s$ of $0.2,0.3,0.4,0.1$. More requests are located at bottom right corner since $r$ has the greatest value among the four parameters.

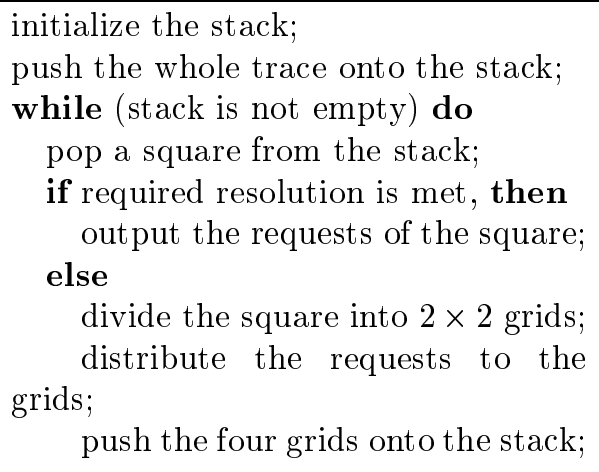

Figure 4: $P Q R S$ trace generation

The above algorithm assumes the same order of $p, q, r, s$ is used in all the levels. A random PQRS trace can be generated by imposing a different order at each step.

\subsection{Parameter Fitting}

The recursive construction algorithm guarantees that PQRS traces have linear entropy plots. The burstiness and spatio-temporal correlation are stable because all the steps use the same parameters to distribute the requests.

Lemma 2 Traces generated by the PQRS model have stable burstiness and correlation as they have 
linear entropy plots.

$$
\left\{\begin{array}{l}
H_{T}^{(n)}=n H_{T}^{(1)} \\
H_{S}^{(n)}=n H_{S}^{(1)} \\
H_{T, S}^{(n)}=n H_{T, S}^{(1)}
\end{array}\right.
$$

Lemma 3 For a PQRS trace generated with parameter $p, q, r, s, p+q+r+s=1$, the entropy rates are

$$
\left\{\begin{array}{l}
R_{T}=-(p+q) \log _{2}(p+q)-(r+s) \log _{2}(r+s) \\
R_{S}=-(p+r) \log _{2}(p+r)-(q+s) \log _{2}(q+s) \\
R_{T, S}=-p \log _{2} p-q \log _{2} q-r \log _{2} r-s \log _{2} s
\end{array}\right.
$$

All the proofs are omitted from the paper for brevity. Equation 8 suggests that $p+q$ determines the temporal burstiness of the synthetic traces and $p+r$ determines the spatial burstiness. Given the same temporal and spatial burstiness, varying the value of $p$ changes the degree of the spatiotemporal correlation.

The parameter fitting algorithm for the PQRS model is simple. For a given trace, plugging the slopes of the entropy plots in Equation 8 gives the values for $p, q, r, s$.

The following two lemmas give some additional features of the PQRS model.

Lemma 4 The Poisson model is a special case of the PQRS model where $p=q=r=s=0.25$.

Lemma 5 The $i$-model is a special case the PQRS model where $\frac{p}{q}=\frac{r}{s}$.

\subsection{Complexity}

The computational complexity of the algorithm is an important property of the model. One would rather choose to collect real traces if the trace generation is too slow. Ideally, the model should offer linear scalability. This section analyzes the computational complexity of the PQRS model. Our analysis shows that both the trace generation and the parameter fitting algorithms offer linear scalability.

Lemma 6 The computation complexity for trace generation in the PQRS model is $O(M \times N)$, where $M$ is the total number of requests and $N$ is the resolution level.

Proof: Omitted for brevity. 
We outline the proof here. We upper-bound the trace generation through a naive implementation of the algorithm. The recursive generation conceptually forms a quad tree. (See Figure 3 (a).) The $4^{n}$ grids in step $n$ form the $4^{n}$ nodes at level $n$ in the quad tree. In the naive implementation, we decide the value of $t$ and $s$ for a request $(t, s)$ by walking down the quad tree from the root to a leaf node. The probability that the request goes into the four subtrees is given by $p, q, r, s$. Enumerating all the requests gives the final trace. The number of levels of the quad tree is bound by $O(N)$, where $N$ is the number of steps involved. Therefore, the complexity of the trace generation is $O(M \times N)$. In reality, $N$ is usually the logarithm to the length of the trace in time (or space).

Similarly, the computation effort of the entropy plots scales linearly to the number of requests as well.

Lemma 7 The computation complexity for parameter fitting algorithm of the PQRS model is $O(M \times N)$.

\section{Proof: Omitted for brevity.}

We give a sketch of the proof here. For a given trace of $M$ requests, the number of points in the entropy plot is $O(N)$. The number of non-zero grids in each step is less than $M$, thus, it takes $O(M)$ computations to generate a point in the entropy plot. Therefore, the total computational complexity to compute the entropy plots is $O(M \times N)$.

In summary, the strength of the PQRS model lies in its power as well as in its simplicity. The model generates traces with stable burstiness and correlation as the real traffic data exhibits. Additionally, the model offers linear scalability.

\section{Experiments}

We evaluate the PQRS model using both disk and memory reference traces. The experiments examine the validity of the PQRS model and compares the performance behavior of the PQRS model traces to the real ones.

We made two main observations. First, the real traffic data have reasonably linear entropy plots which verifies the assumption we made in the PQRS model. Second, strong spatio-temporal correlation plays an important role in performance behavior and invalidates the i-model. The PQRS model, on the other hand, leads to performance measures that match reality. 


\subsection{Experiment Setup}

Table 2 gives the summary of the disk I/O and memory traces in use.

Cello disk traces. The disk traces were collected on a UNIX file server in HP on June 12th, 1992 [13]. The server has 8 disks attached to it. Total of six traces are in use: Disk-a for the aggregation of all the disk requests, Disk-r for all the read requests, Disk-w for all the write requests, and Disk-0, Disk-2, Disk-7 for individual disk 0, 2, 7. All the traces are one day long. The other five disks are not studied because of the small volume of disk requests. The arrival time is accurate up to microseconds. The disk block number ranges from 0 to more than 5,000,000.

TPC-C memory reference traces. The TPC-C memory traces were collected on a realistic processor simulator running TPC-C workloads on Shore [3]. TPC-C [1] is an online transaction processing (OLTP) benchmark modeling the order processing operations of a wholesale supplier. There are total of six traces: five for five types of transactions and one for a mixture of different types of transactions. Only references to the heap area are studied here.

Evaluation tools. The ultimate goal of traffic modeling is to facilitate system designs. Therefore, we focus on the performance aspect of the traces. We use the response time and queue length distributions for disk traces and the cache miss ratio for memory reference traces as our performance metrics.

Methodology. We want to answer the following questions: (a) Does real traffic have stable burstiness and correlation over aggregation? The real traffic should have linear entropy plots for the PQRS model to work. (b) If so, how does the PQRS model perform in modeling them? Ideally, the synthetic traces should have the same performance behavior as the real ones when the parameters used in trace generation are derived from the real ones.

\subsection{Model Checking}

The PQRS model is designed for the traffic data with stable burstiness and spatio-temporal correlation. Therefore, the traffic data should have linear entropy plots for the PQRS model to work. 
At the same time, linear entropy plots give a good estimation for parameter $p, q, r, s$.

Figure 5 shows the entropy plots for the disk and memory traces. We have made the following observations.

1. The entropy plots are reasonably linear, suggesting stable burstiness and correlation in the traces. The estimated slopes are listed in Table 2. The stability ensures that the traces are well within the capability of the PQRS model.

2. Strong spatio-temporal correlation exists in both types of traces. The mutual information ranges from 0.313 to 0.696 for disk traces and from 0.166 to 0.417 , indicating strong correlation.

3. The PQRS model is able to model uniform traces as well. $R_{T}$ for the memory traces is close to 1 , suggesting a uniform distribution of the memory accesses on time. This is because the program is consistently accessing data during its course of execution.

In summary, real traffic data has stable burstiness and correlation over aggregation and is within the capability of the PQRS model. Strong correlation exists, invalidating the independence assumption of the i-model.

Table 2 gives the estimated $p, q, r, s$ value from Equation (8) for the real traces. The following sections compare the performance behavior of the real traces and the PQRS traces generated from the estimated $p, q, r, s$ value.

\subsection{Disk Trace Evaluation}

Figure 6 show the response time and queue length distributions of the real and the PQRS traces on a realistic disk simulator [8]. Both distributions are in negative cumulative form and in log-scale. That is, a point $(10,0.01)$ in the response time distribution plot says the more than $1 \%$ of the disk requests have response time greater than 10 milliseconds. We expect that traces with strong spatiotemporal correlation should have short tails in these distributions as requests close in location can be served faster.

The comparison has shown that the PQRS traces, accurately capturing the burstiness and the correlation, simulates the performance behavior of the real traces very well. 


\begin{tabular}{|c|r|r|r|r|r|c|}
\hline Trace & Total disk requests & $\hat{R}_{T}$ & $\hat{R}_{S}$ & $\hat{R}_{T, S}$ & $I_{T, S}$ & $(\hat{p}, \hat{q}, \hat{r}, \hat{s})$ \\
\hline Disk-a & $4,575,798$ & 0.641 & 0.819 & 1.058 & 0.402 & $(0.862,0.001,0.257,0.741)$ \\
\hline Disk-r & $1,822,781$ & 0.847 & 0.833 & 0.984 & 0.696 & $(0.016,0.258,0.720,0.006)$ \\
\hline Disk-w & $3,300,628$ & 0.641 & 0.728 & 0.992 & 0.377 & $(0.150 .0 .013,0.053,0.784)$ \\
\hline Disk-0 & $1,101,416$ & 0.814 & 0.690 & 0.941 & 0.563 & $(0.043,0.184,0.772,0.001)$ \\
\hline Disk-2 & $1,396,649$ & 0.790 & 0.723 & 0.904 & 0.609 & $(0.200,0.027,0.001,0.772)$ \\
\hline Disk-7 & 371,320 & 0.722 & 0.573 & 0.881 & 0.414 & $(0.056,0.135,0.808,0.001)$ \\
\hline
\end{tabular}

(a) Cello disk trace summary

\begin{tabular}{|c|r|r|r|r|r|r|c|}
\hline Trace & Length & Total requests & $\hat{R}_{T}$ & $\hat{R}_{S}$ & $\hat{R}_{T, S}$ & $I_{T, S}$ & $(\hat{p}, \hat{q}, \hat{r}, \hat{s})$ \\
\hline New Order & $14,990,636$ & $4,000,000$ & 0.962 & 0.200 & 0.996 & 0.166 & $(0.030,0.255,0.001,0.714)$ \\
\hline Payment & $17,242,172$ & $4,573,044$ & 0.963 & 0.281 & 1.042 & 0.202 & $(0.239,0.047,0.713,0.001)$ \\
\hline Order Status & $1,355,168$ & 268,943 & 0.950 & 0.456 & 0.989 & 0.417 & $(0.095,0.185,0.001,0.722)$ \\
\hline Delivery & 525,100 & 129,388 & 0.957 & 0.439 & 0.987 & 0.409 & $(0.090,0.192,0.001,0.717)$ \\
\hline Stock Level & $14,453,440$ & $3,613,360$ & 0.974 & 0.349 & 1.052 & 0.271 & $(0.231,0.064,0.704,0.001)$ \\
\hline Mix & $12,268,876$ & $4,000,000$ & 0.983 & 0.309 & 0.990 & 0.302 & $(0.248,0.054,0.697,0.001)$ \\
\hline
\end{tabular}

(b) TPC-C memory reference trace summary (Trace length in CPU cycles)

Table 2: Trace Summary
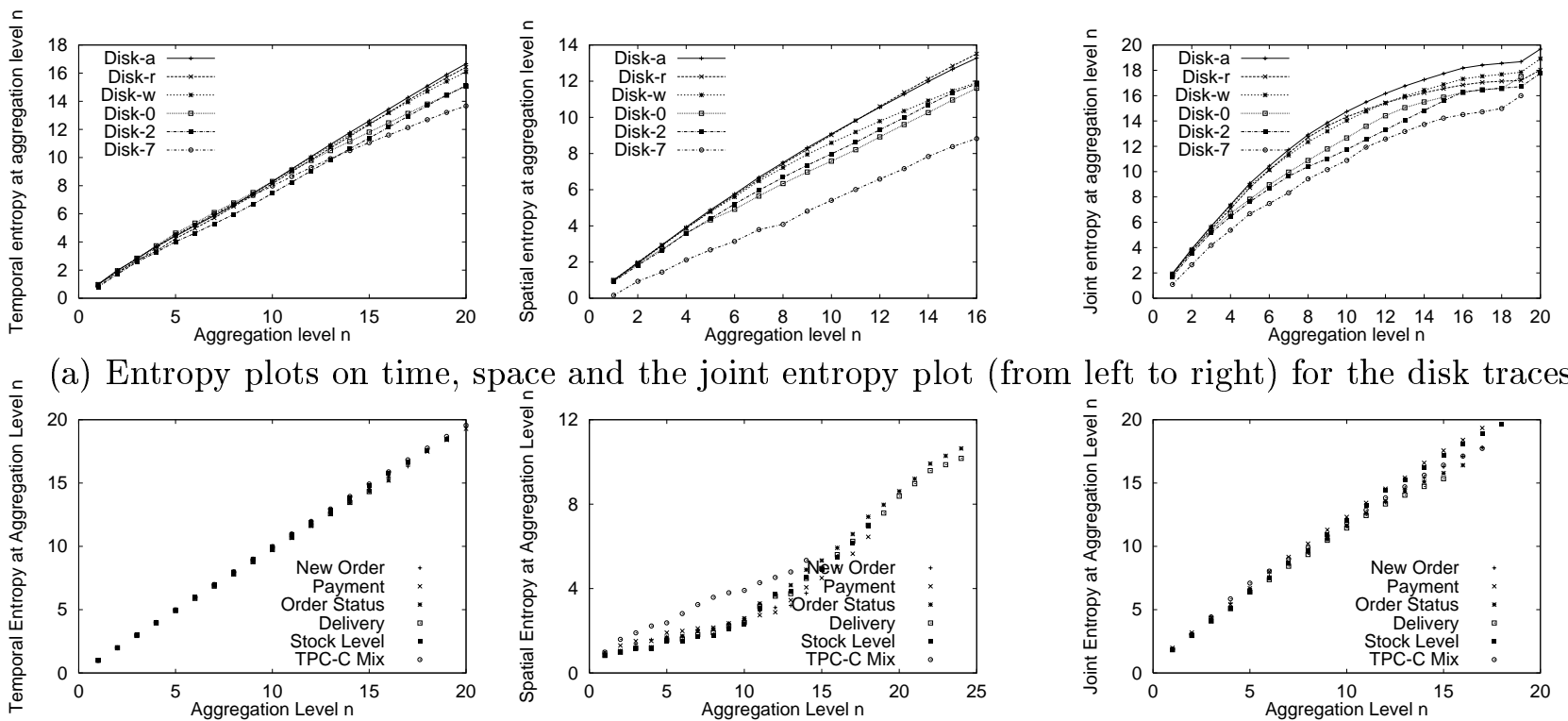

(a) Entropy plots on time, space and the joint entropy plot (from left to right) for the memory traces.

Figure 5: Entropy plots for the cello disk and TPC-C memory reference traces. 


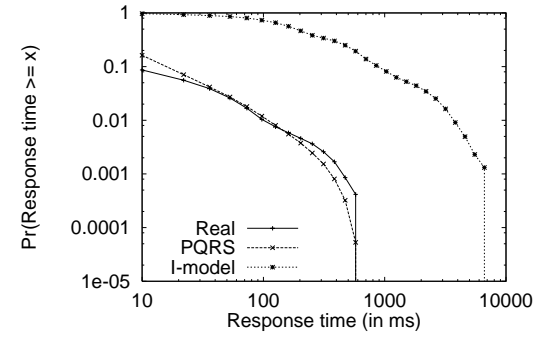

Disk-0

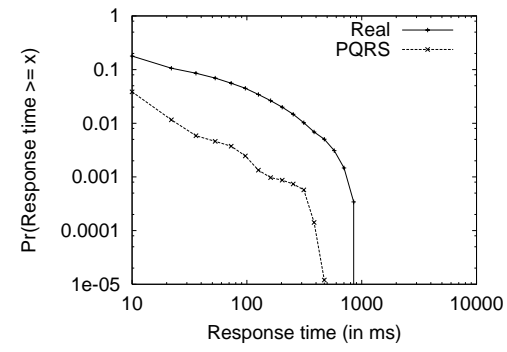

Disk-2

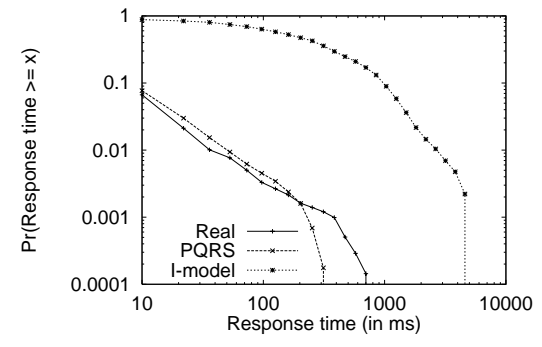

Disk-7

(a) Response time distribution in NCDF

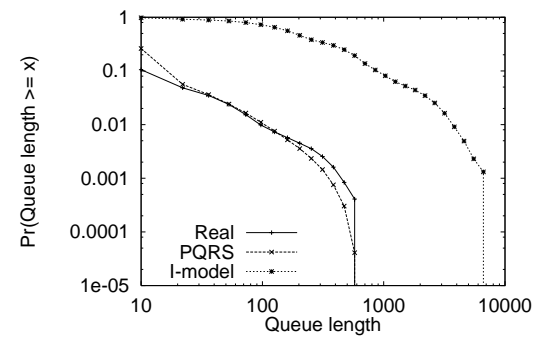

Disk-0

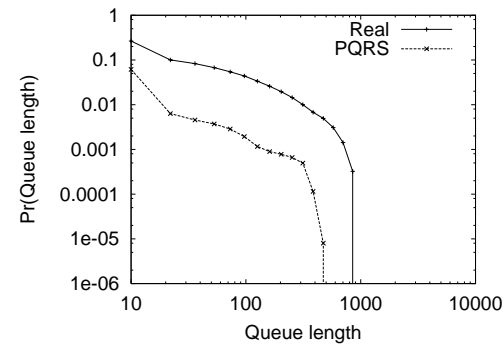

Disk-2

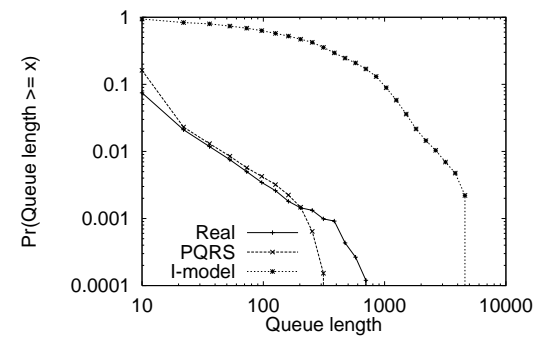

Disk-7

(b) Queue length distribution in NCDF

Figure 6: Disk Trace Performance Evaluation. (I-model traces for disk-2 crashes due to queue saturation.

1. Strong spatio-temporal correlation plays an important role in performance behavior of the traces as we have expected. Traces with strong correlation yield shorter tails in both distributions. The real traces and the i-model traces have exactly the same temporal and spatial burstiness. However, the i-model traces produce extremely large response time because of the independence assumption. The i-model results for Disk-2 are missing because the queue becomes long enough to saturate the system.

2. The PQRS model works amazingly well in simulating the real traffic behavior by accurately capturing both the burstiness as well as the strong correlation at all aggregation levels.

The above comparison has shown that the PQRS model mimic the real disk I/O traffic very well in performance behavior. 


\subsection{Memory Trace Evaluation}

Memory trace evaluation involves comparing the cache miss rates of the real traces to the PQRS traces. Two facts justify our choice of using the cache miss rate to evaluate the performance behavior. First, the miss rate is an important performance metric in computer architecture research. Second, the cache miss rate reflects the spatio-temporal behavior of the trace. Memory references on nearby locations have a better chance to be cache hits if they are close to each other in arrival time. Therefore, strong spatio-temporal correlation leads to low cache miss rates.

Figure 7 compares the cache miss rates for three sets of traces: $\mathrm{R}$ for the real traces; $\mathrm{I}$ for the i-model traces generated from the same marginals as the real ones; P for the PQRS traces with parameter values as listed in Table 2. Six groups of bars show the cache miss rates on six different cache sizes in each graph.

We observe that the traces with high degree of spatio-temporal correlation, such as the $\mathrm{R}$ and $\mathrm{P}$ traces, suffer low cache miss rates as we have expected. The relative error of the PQRS traces is within $30 \%$. On the other hand, the I traces, assuming independence between time and space, experience extremely high miss rates and have relative error as high as $1800 \%$.

\subsection{Summary}

Both disk traces and memory references traces have shown reasonably stable burstiness and spatiotemporal correlation over aggregation as suggested by the linear entropy plots. Strong correlation between the arrival time and location of the requests exists in both types of traffic data and it has a significant impact on the performance behavior of the traces. Therefore, traffic modeling should take spatio-temporal correlation into consideration.

The PQRS model, carefully designed for this type of traffic data, is able to replicate the behavior of real traces as shown by the experiments. The i-model, on the other hand, failed to do so by ignoring the correlation.

\section{Conclusions}

Modeling disk traffic is a hard problem [8], especially when we need to capture both the temporal as well as spatial correlations. The contributions of this paper are the following: 

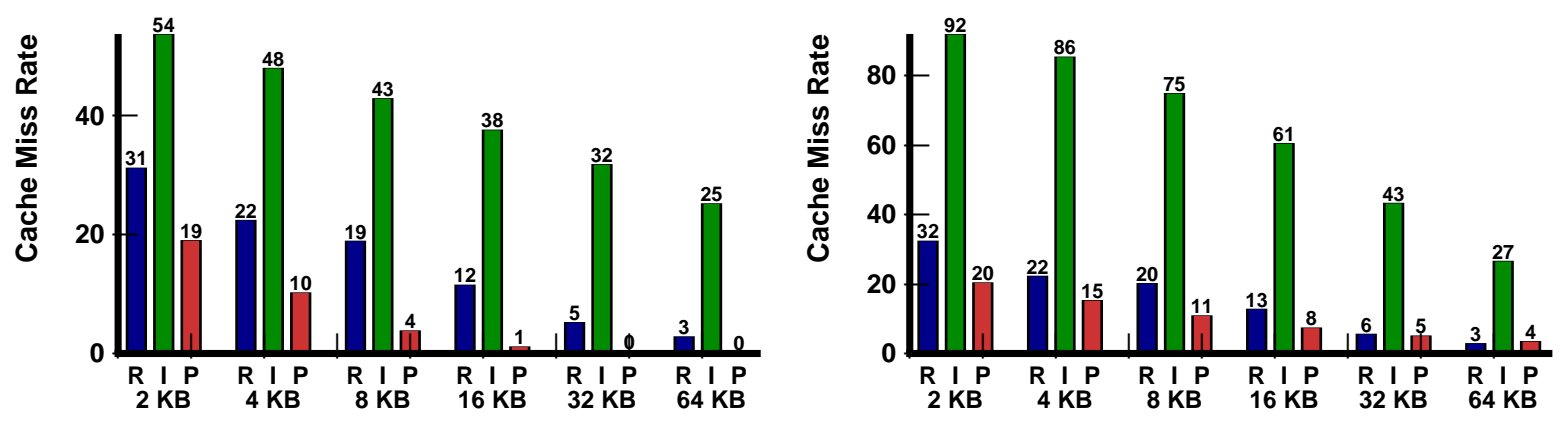

(a) New Order Transaction

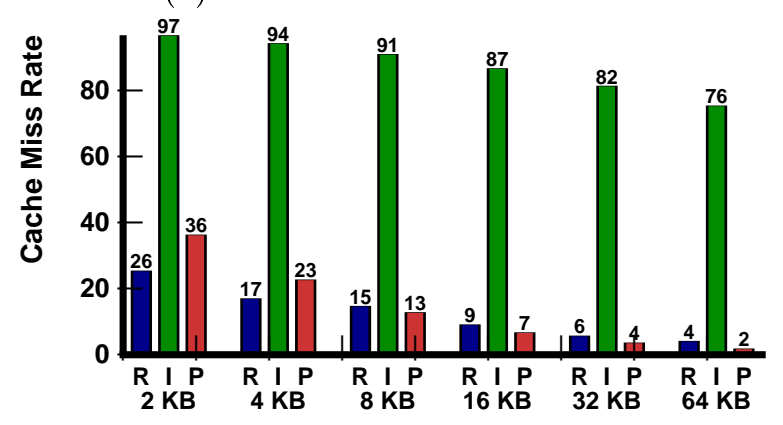

(b) Payment Transaction

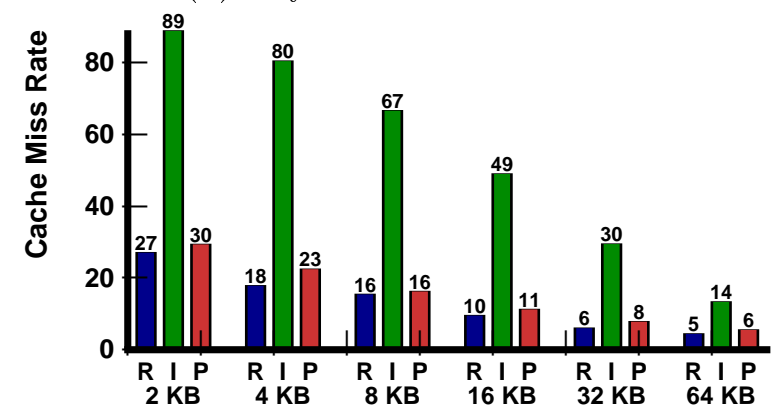

(c) Order Status Transaction
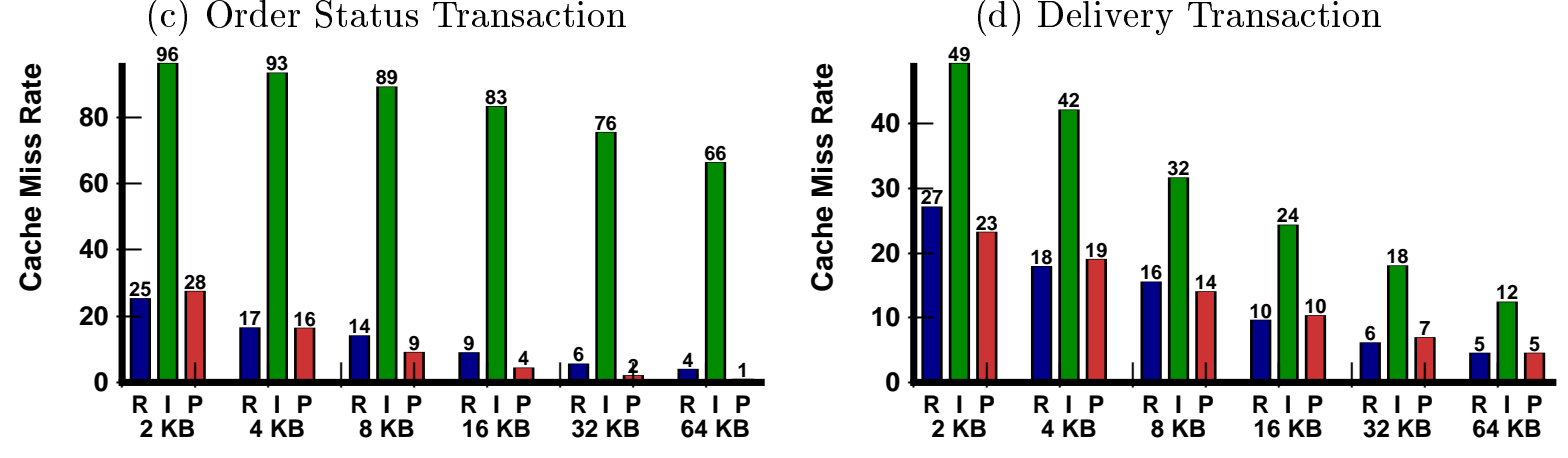

(e) Stock Level Transaction

(f) TPC-C Transaction Mix

Figure 7: Comparison of the performance behavior of the memory reference traces. Three types of traces are evaluated against the a realistic cache simulator. Trace " $R$ " stands for "Real"; " $I$ " for the i-model, and "P" for the PQRS model. 
- we propose a simple model, the PQRS-model, that achieves all goals: it can be bursty or uniform in time, bursty or uniform in space, and it can give zero to $100 \%$ correlation between space and time.

- we propose a way to measure the spatio-temporal correlation, through the entropy plots and the mutual information plots, which also showed that the burstiness and correlations remain stable for many scales.

Smaller contributions include

- we are the first to quantify the popular, but vague intuition that memory and disk accesses exhibit locality, not only in space or time, but in space-time as well.

- we give fast, scalable algorithms to build and run our model: They require linear time on the number of requests $M$, to estimate the model parameters, and linear time again $(O(M))$ in generating a trace of $M$ requests.

- experiments on multiple real datasets show that the simple PQRS model can mimic them very well, leading to good performance predictions (cache-hit-ratios, queue length distributions). In contrast, the only other competitor, the independence model (i-model), fails miserably. For example, the PQRS model traces have mean error $30 \%$ compared to the real traces while the independence assumption can have error as high as $1800 \%$ in cache miss rates for the memory traces.

One promising research direction could focus on the use of the PQRS model for other spatiotemporal settings (e.g., earthquakes over space and time). Another direction could be the analytical derivation of performance measures of interest (like the cache-hit ratio, or disk queue length distributions), given the $p, q, r, s$ values of a trace.

Acknowledgement We wish to thank John Wilkes from HP Storage System Lab for providing us with the cello disk trace and constant feedback on our work. We are grateful to Jiri Schindler for helping on the disk simulator. In particular, we appreciate Prof. Anthony Brockwell for his insight on classical statistical models. 


\section{References}

[1] TPC-C benchmark specification. http://www.tpc.org/.

[2] Paul Bardord and Mark Crovella. Generating representative web workloads for network and server performance evaluation. In SIGMETRICS'98, pages 151-160, 1998.

[3] M. Carey, D. J. DeWitt, M. Franklin, N. Hall, M. McAuliffe, J. Naughton, D. Schuh, M. Solomon, C. Tan, O. Tsatalos, S. White, and M. Zwilling. Shoring up persistent applications. In SIGMOD, 1994.

[4] Stavros Christodoulakis. Implications of certain assumptions in database performance evaluation. $A C M$ Transactions on Database Systems, 9(2):163-186, 1984.

[5] David R. Cox and Valerie Isham. Point processes. Chapman and Hall, 1980.

[6] Noel A. C. Cressie. Statistics for spatial data. J. Wiley, 1991.

[7] Mark E. Crovella and Azer Bestavros. Self-similarity in world wide web traffic evidence and possible causes. In Proc. of the 1996 ACM SIGMETRICS Intl. Conf. on Measurement and Modeling of Computer Systems, May 1996.

[8] Gregory R. Ganger. Generating representative synthetic workloads: An unsolved problem. In Proceedings of the Computer Measurement Group (CMG) Conference, 1995.

[9] Mark W. Garrett and Walter Willinger. Analysis, modeling and generation of self-similar VBR video traffic. In SIGCOMM'94, 1994.

[10] María E. Gómez and Vicente Santonja. Self-similiarity in I/O workload: Analysis and modeling. In Workshop on Workload Characterization, 1998.

[11] W. E. Leland, M. S. Taqqu, W. Willinger, and D. V. Wilson. On the self-similar nature of ethernet traffic (extended version). IEEE Transactions on Networking, pages 1-15, 1994.

[12] Ruldolf H. Riedi, Matthew S. Crouse, Vinay J. Ribeiro, and Richard G. Baraniuk. A multifractal wavelet model with application to network traffic. In IEEE Transactions on Information Theory, number 3, April 1999.

[13] Chris Ruemmler and John Wilkes. Unix disk access patterns. In Proceedings of the Winter'93 USENIX Conference, pages 405-420, 1993.

[14] Claude E. Shannon and Warren Weaver. Mathematical Theory of Communication. University of Illinois Press, 1963.

[15] Elizabeth Shriver, Arif Merchant, and John Wilkes. An analytic behavior model for disk drives with readahead caches and request reordering. In SIGMETRICS'98, 1998.

[16] Mengzhi Wang, Tara Madhyastha, Ngai Hang Chan, Spiros Papadimitriou, and Christos Faloutsos. Data mining meets performance evaluation: Fast algorithm for modeling bursty traffic. In ICDE'02, 2002 . 